\title{
Graphene and Boron Nitride-Based Nanocomposites with Enhanced Thermal Properties
}

\author{
Pradip Majumdar $^{1 *}$ and Amartya Chakrabarti ${ }^{2,3}$ \\ ${ }^{1}$ Department of Mechanical Engineering, Northern Illinois University, USA \\ ${ }^{2}$ Department of Physical Sciences, Dominican University, River Forest, USA \\ ${ }^{3}$ Applied Material Systems Engineering Inc., Streamwood, USA
}

\begin{abstract}
Polymer composites with a high thermal conductivity are always desired for different applications. Improved thermal conductivity of polymers can be obtained via dispersion of metal particles in a polymer matrix; however a good dispersion and thermal coupling cannot be achieved. We have designed and developed a formulation with enhanced thermal conductivity of silicone and epoxybased resin systems using graphene and boron nitride based nanomaterials synthesized in our laboratories. The nanocomposites are characterized thoroughly and excellent thermal conductivity improvement was observed. A detailed data analysis with different characterization techniques will be discussed and demonstrated.
\end{abstract}

Keywords

Few-layer graphene, Boron nitride nanotube, Polymer nanocomposite, Thermal conductivity

\section{Introduction}

Polymers typically suffer issues with Low thermal conductivities. However, many important applications of polymer require them to be able to be thermally conductive so that they can compete with metals or ceramics for use in thermal management systems. In previous efforts, Thermal Interface Materials (TIMs) based on Carbon Nanotubes (CNTs) suffered from Kapitza or thermal contact resistance and failed to provide the anticipated performance [1]. Moreover, at least 20\% Multiwall Carbon Nanotubes (MWNTs) were needed to be mixed with $80 \%$ graphene platelets as filler for the TIMs in order to gain the required integrity. The graphene nanoplatelets were the early versions of graphitic graphene with 10 to 20 layers. Re-centre ports from Balandin, et al. showed that graphene with appropriate aspect ratio can help in reduction of Kapitza resistance and promote thermal coupling in graphene flake and matrix materials to increase effective thermal conductivity [2].

Boron Nitride Nanotubes (BNNTs) also exhibit very high thermal conductivities [3]. However, large scale production of BNNTs remained a major challenge to the scientific community as well as industries [4]. The most commonly use methods for preparation of BNNTs include ball milling and annealing, $[5,6]$ arc discharge, [7,8] chemical vapor deposition, [9-11] laser ablation, [12] mechano-thermal [13] and other chemical syntheses techniques [14,15]. Many of them use drastic reaction conditions e.g., high reaction temperature (in the range of $1000-1400^{\circ} \mathrm{C}$ ) and needed expensive and complicated reaction setups. Therefore, a simple way of manufacturing large scale BNNTs is warranted.

In this work, we have used few layer graphene and boron nitride nanomesh as two primary candidates to be

*Corresponding author: Pradip Majumdar, Department of Mechanical Engineering, Northern Illinois University, DeKalb, IL 60510, USA, E-mail: pdmajumdar@gmail.com

Received: June 21, 2017: Accepted: November 01, 2017: Published: November 03, 2017

Copyright: (C) 2017 Majumdar P, et al. This is an open-access article distributed under the terms of the Creative Commons Attribution License, which permits unrestricted use, distribution, and reproduction in any medium, provided the original author and source are credited. 
Table 1: General comparison between the techniques of graphene production.

\begin{tabular}{|l|l|l|}
\hline & $\begin{array}{l}\text { Chemical } \\
\text { exfoliation }\end{array}$ & $\begin{array}{l}\text { Dry-ice } \\
\text { method }\end{array}$ \\
\hline Uses renewable feed stocks & No & Yes \\
\hline Environmentally benign & No & Yes \\
\hline Sustainable & No & Yes \\
\hline Low energy requirements & No & Yes \\
\hline $\begin{array}{l}\text { Scalable to industrial } \\
\text { production levels }\end{array}$ & Yes & Yes \\
\hline Quality of the product & Medium & High \\
\hline
\end{tabular}

in corporate in the selected polymer matrix and thermal conductivity was measured to ensure the improvement of the thermal properties. Boron Nitride Nanomesh (BNNM) is defined as a mixture of nanotubes and nanoflakes in a variety of ratio ranging from 1:1 to 1:2 nanotubes and nanoflakes, respectively.

The most common method of graphene fabrication is the exfoliation of graphite [16]. Exfoliation involves use of strong oxidizing agents such as sulfuric acid and/or potassium permanganate. Graphite is a limited natural resource, and very recently China, the world's largest producer and exporter of graphite, set access standards for its graphite industry [17]. Besides being tedious and environmentally challenging, the mechanical exfoliation process for graphene production is going to be very expensive due to restrictions on the usage of the starting material graphite. Supply chain disruptions area distinct possibility.

Researchers at Northern Illinois University have been successfully produced few- layer graphene using an ovel methodology now being termed as dry-ice method [18]. The dry-ice method does not use any graphite nor does it involve any toxic or harmful chemicals (Table 1). The innovative and cost-effective methodology of burning magnesium in dry-ice is safer, faster and cleaner than other methods which involve hazardous materials. Moreover, the combustion of the magnesium metal in gaseous $\mathrm{CO}_{2}$ helps to form few-layer graphene. During the reduction process, the retention time of the carbon atoms formed is not significantly long to produce multi-layer graphene and the product is thus restricted between 3 and 7 layers. None the less, the simple nature of the chemistry involved suggests that scale-up will be very straight forward since the chemical processes are deceptively simple and easy to control. This has enabled the production of few-layer graphene in industrial scale quantities. Furthermore, the crystalline nature of the graphene sheets produced by the dry-ice method should be electrically conductive while the smaller dimension of the nanosheets may facilitate an easy dispersion in solvents.

\section{Materials and Methods}

\section{Few-layer graphene}

Few-layer graphene was synthesized using a further modification of the above mentioned process in collaboration with Applied Material Systems Engineering Inc. The current synthetic approach depended upon achieving more complete burning of magnesium and smooth retention of flame propagation. The most common difficulty, encountered during magnesium burning inside the dry ice cubes, was the random fire quenching with in complete ignition of the metal. Abrupt and rapid quenching is observed that provides only small feature sizes for FLGs. The Self-Sustaining Magnesio-Thermic Combustion (SSMTC) process, involving magnesium burning, has been successfully employed to produce semi conducting carbides on an industrial scale [19]. Following similar protocol in the self-sustaining combustion front propagation defect-free graphene with larger sizes has been currently produced. The smooth and complete burning with appropriate quenching was achieved to develop Few-Layer Graphene (SSMTC-FLG).

\section{Boron Nitride Nanomesh (BNNM)}

A method has been developed to prepare hexagonal boron nitride nanomesh in a relatively lower temperature and high yield [20]. The pyrolysis of boron and nitrogen containing precursors in the presence of certain catalysts in a sealed autoclave produced BNNMs in large quantities. Optimization of the reaction condition in terms of temperature and time of reaction is currently underway.

\section{Polymer matrix}

Polymeric materials used for the nanocomposite formulation are processed with appropriate nanofillers developed and designed in our laboratories. The PDMS silicone, and the cycloaliphatic epoxy based resins are commercially available and they were chosen as they meet NASA out gassing requirements. The silicone formulations were prepared by AMSENG based on the Gelest materials which were further processed at AMSENG for meeting the low out gassing needs. We have used solution casting method, published in literatures, to produce the nanocomposites [21]. A solution was prepared using the nanomaterials and the polymer matrix in suitable solvents, which was then casted on an aluminum substrate and dried under vacuum to produce the nanocomposites. However, these formulations have not been perfected yet for the shelf life issues, whereas the epoxy materials were obtained from the Master Bond Inc.

\section{Results and Discussion}

\section{Few-layer graphene}

The products graphene were characterized by Raman spectroscopy (Renishaw in Via Raman microscope, 532 $\mathrm{nm}$ laser source) to identify the form of the carbon allotrope. The Raman signature peak for any carbon allotrope are around 1350, 1580 and $2700 \mathrm{~cm}^{-1}$ and are la- 
beled the D, G, and G' (or 2D) band peaks, respectively. Depending on the intensity, width, and number of splits in a peak, the carbon sample can be determined to be single layer, bilayer, few-layer or graphite. Raman spectroscopy is considered to be extremely sensitive for detecting variation in the number of graphene layers with an increase in the number of layers resulting in a blue shift for the G' peak along with peak broadening and a

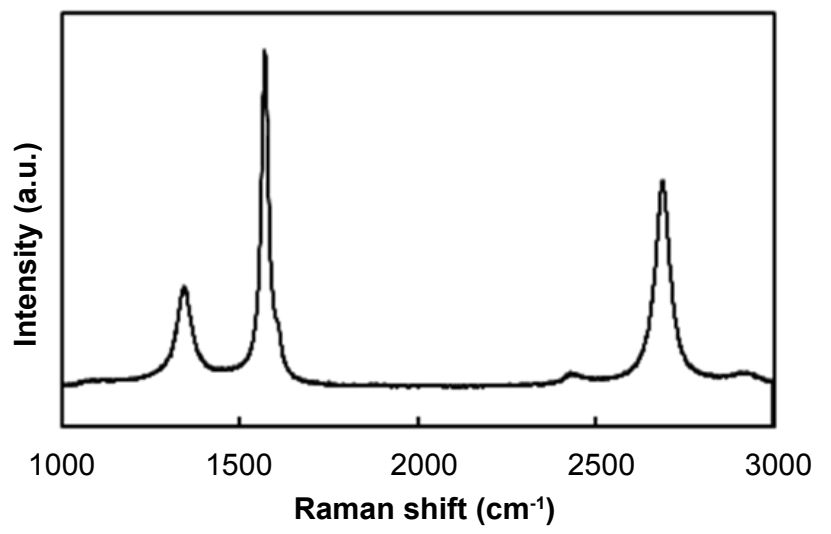

Figure 1: Raman spectroscopy of FLGs. decrease in intensity. The peaks for FLGs are observed at $2685 \mathrm{~cm}^{-1}$ (G' band), $1571 \mathrm{~cm}^{-1}$ (G band) and $1336 \mathrm{~cm}^{-1}$ (D band) (Figure 1) and are typical for few-layer graphene with fewer than 4 layers [13].

TEM (Hitachi H-600) was utilized to determine product morphology. TEM imaging of untreated mixtures clearly showed fused magnesium oxide cubes (Figure 2a) as anticipated, and these are thought to be the reason for the formation of larger graphene flake size. Figure $2 \mathrm{~b}$ and Figure $2 \mathrm{c}$ exhibit FLGs of large dimension, typically within the range of $500 \mathrm{~nm}$ to a few micron. On the other hand, HRTEM (JEOL JEM-2100F, Figure 3) clearly shows the number of layers of graphene materials formed during the process. Although the major fraction of the product was determined to be comprised of FLGs with less than 4 layers, a fraction of the product was found to have more than 5 layers. Application of powerful sonication showed that the product can be dispersed resulting in finer dimensions with fewer numbers of layers; this indicates the potential for introducing sonication and separation in to the production process and the need for further development.


Figure 2: TEM image of the a) Untreated product, as retrieved after burning; b) \& c) FLGs.


Figure 3: HRTEM images of FLGs with the number of layers between a) 3 and b) 7 . 

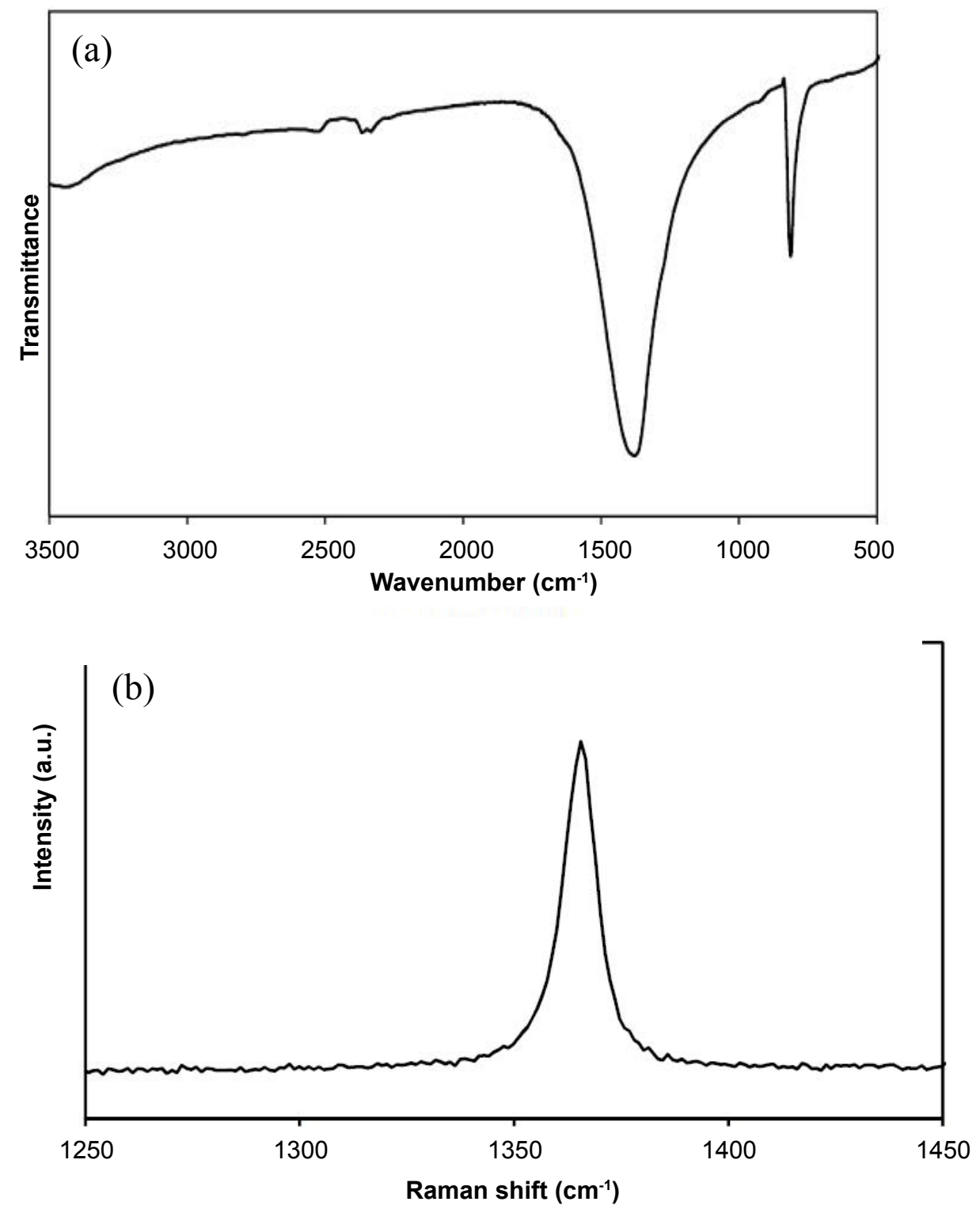

Figure 4: a) FT-IR; b) Raman spectrum of BNNT samples.

In a summary, SSMTC-FLGs purified and separated by established practices [18] were shown to be typically 3 layer graphene in larger flakes dimensions based on imaging carried out on several samples as recommended [3]. The present method produces a min or fraction of FLGs that are equivalent to those produced in earlier efforts [3] with 3 to 7 layers, but the major fraction has fewer than 4 layers and typically 3 layers with large flake size of several hundred nanometers.

\section{Boron nitride nanomesh}

Bonding patterns of the $\mathrm{B}$ and $\mathrm{N}$ atoms in nanotubes and purity of the products were further verified by FT-IR (and Raman spectroscopy (Figure $4 \mathrm{a}$ and Figure $4 \mathrm{~b}$ ). The FT-IR peak at $1380 \mathrm{~cm}^{-1}$ is due to the stretching vibration of B-N bonds. A comparatively weaker peak at 812 $\mathrm{cm}^{-1}$ can be attributed to the bending vibration of $\mathrm{B}-\mathrm{N}-\mathrm{B}$ bonds of Hexagonal Boron Nitride (h-BN). Raman spectroscopy of the BNNT samples was performed with a 532 $\mathrm{nm}$ laser source. A very intense sharp peak at $1365 \mathrm{~cm}^{-1}$ acts as the definite confirmation of the presence of h-BN in the sample.

Morphology of the product has been determined via Scanning Electron Microscopy (SEM). While Transmission Electron Microscopy (TEM) helps to observe a sample in two-dimension, SEM provides a three-dimensional rendering. SEM not only gives us information on the surface and structure of the samples, but also it helps to examine a larger area of the samples. Thus, uniformity in structures and homogeneity of the products can be confirmed. The BNNT product has shown abundant nanotubes to be present in the compound over abroad area of the sample and he open ends of the nanotubes became distinctly visible (Figure 5a). The average dimension of 


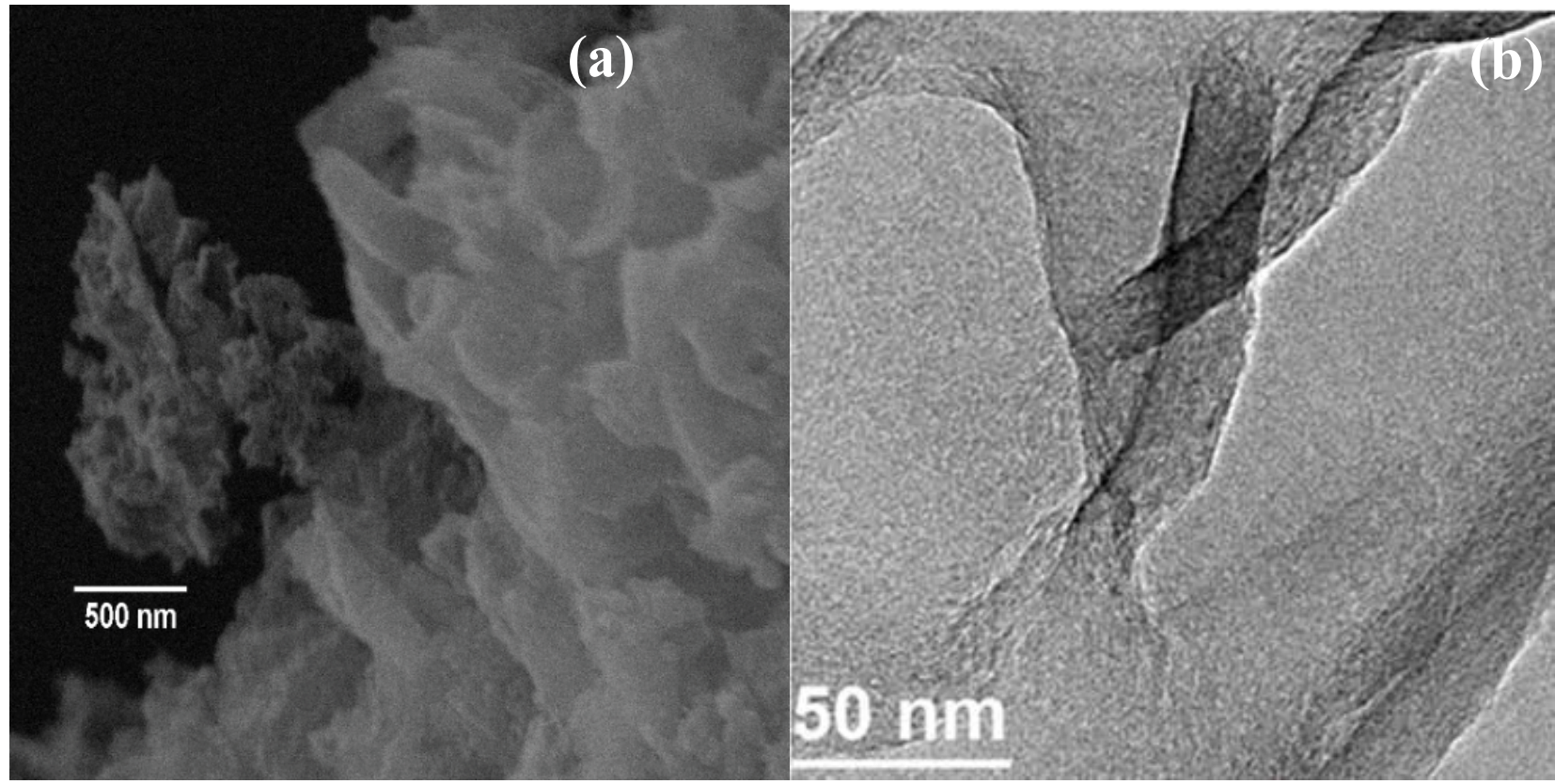

Figure 5: a) SEM image; b) TEM image BNNM samples.

Table 2: Effective thermal conductivity data for PDMS (S6)-SSMTC-FLG and LOE poxy(UV-30)-SSMTC-FLG composites [22].

\begin{tabular}{|c|c|c|c|}
\hline Sample & Pressure (psi) & Temperature $\left({ }^{\circ} \mathrm{C}\right)$ & Thermal conductivity $[\mathrm{W} /(\mathrm{m} . \mathrm{K})]$ \\
\hline \multirow{2}{*}{$\begin{array}{l}\text { PDMS S6 + } 2 \% \text { SSMTC-FLG } \\
\text { (Al Face sheet, } t=6.56 \mathrm{~mm} \text { ) }\end{array}$} & 40 & 24.2 & 2.761 \\
\hline & 40 & 52.55 & 2.838 \\
\hline \multirow{2}{*}{$\begin{array}{l}\text { PDMS S6 + } 2 \% \text { SSMTC-FLG } \\
\text { (Al Face sheet, } t=6.41 \mathrm{~mm} \text { ) }\end{array}$} & 40 & 28.75 & 2.132 \\
\hline & 40 & 56.72 & 2.224 \\
\hline PDMS S6 (Al Face sheet, $\mathrm{t}=6.40 \mathrm{~mm}$ ) & 40 & 28.25 & 0.246 \\
\hline \multirow[t]{2}{*}{ LOE poxyUV-30 (AI Face sheet) } & 40 & 20.95 & 0.202 \\
\hline & 40 & 50.48 & 0.255 \\
\hline \multirow{2}{*}{$\begin{array}{l}\text { LOE poxyUV-30 + } 10 \% \\
\text { SSMTC-FLG (AI Face sheet) }\end{array}$} & 40 & 22.75 & 2.356 \\
\hline & 40 & 51.7 & 2.454 \\
\hline
\end{tabular}

Table 3: Effective thermal conductivity data for PDMS (S6)-BNNM and LOE poxy(UV-30)-BNNM composites [22].

\begin{tabular}{|c|c|c|c|}
\hline Sample & Pressure (psi) & Temperature $\left({ }^{\circ} \mathrm{C}\right)$ & Thermal conductivity $[\mathrm{W} /(\mathrm{m} . \mathrm{K})]$ \\
\hline \multirow{2}{*}{$\begin{array}{l}\text { PDM SS6 + 4\%-BNNM } \\
\text { (Al Face sheet, } t=6.56 \mathrm{~mm} \text { ) }\end{array}$} & 40 & 24.0 & 1.763 \\
\hline & 40 & 51.45 & 1.838 \\
\hline \multirow{2}{*}{$\begin{array}{l}\text { PDMS S6 + 4\% SSMTC-FLG } \\
\text { (Al Face sheet, } t=6.41 \mathrm{~mm} \text { ) }\end{array}$} & 40 & 28.75 & 1.132 \\
\hline & 40 & 56.72 & 1.224 \\
\hline PDMS S6 (Al Face sheet, $\mathrm{t}=6.40 \mathrm{~mm}$ ) & 40 & 28.25 & 0.246 \\
\hline \multirow[t]{2}{*}{ LOE poxyUV-30 (AI Face sheet) } & 40 & 20.95 & 0.202 \\
\hline & 40 & 50.75 & 0.255 \\
\hline \multirow{2}{*}{$\begin{array}{l}\text { LOE poxyUV-30 + } 6 \% \text { BNNM } \\
\text { (Al Face sheet) }\end{array}$} & 40 & 21.75 & 1.356 \\
\hline & 40 & 52.75 & 1.454 \\
\hline
\end{tabular}

the nanostructures was determined to be around $800 \mathrm{~nm}$.

TEM characterization was conducted on the BNNM samples and the data is consistent with the findings from SEM imaging (Figure 5b).

The nanocomposite material systems were further evaluated in these R\&D efforts for its performance for the thermal coupling with typical polymeric system of interest. It was observed that both the SSMTC-FLG and
BNNM samples disperse nicely in the chosen polymer with the help of such solvents. Lock heed Martin Corporation and United Technology's Sundstrand Division assisted us for measuring the thermal conductivity using steady state method and measurement techniques. The thermal conductivity measured is tabulated below in Table 2 and Table 3. The conductivity data clearly exhibited an improvement in thermal conductivity as we observed a ten-fold increment in the conductivity values compared 
to the base polymers used as controls. The conductivity enhancement can be attributed to the thermal coupling between the nanomaterials and polymer interfaces [22].

\section{Conclusions}

In our present work, we have demonstrated preparation of few-layer graphene and hexagonal boron nitride nanomesh and their effectiveness of thermal coupling with selected polymeric systems were evaluated. Both the processed SSMTC-FLG and BNNM were added to the space grade low out gassing polymers for observation of thermal enhancement in polymers. The selected LOPDMS had thermal conductivity of the handbook value of $0.25 \mathrm{~W} /(\mathrm{m} . \mathrm{K})$, and LO-Epoxy had the handbook value of $0.15 \mathrm{~W} /(\mathrm{m} . \mathrm{K})$. Graphene in corporation has shown excellent improvement of thermal conductivity of the polymers with at en-fold increment. BNNM also has shown significant property enhancement. Our data clearly exhibits the effectiveness of such composites in a variety of thermal applications, including thermal interface materials.

\section{References}

1. Singh D, Murthy JY, Fisher TS (2011) Mechanism of Thermal Conductivity Reduction in Few-Layer Graphene. Journal of Applied Physics 110: 044317.

2. Balandin AA, Ghosh S, Bao W, Calizo I, Teweldebrhan D, et al. (2008) Superior Thermal Conductivity of Single-Layer Graphene. Nano Lett 8: 902-907.

3. Chang C, W-Q Han, Zettl A (2005) Thermal conductivity of B-C-N and BN nanotubes. Journal of Vacuum Science \& Technology B, Nanotechnology and Microelectronics: Materials, Processing, Measurement, and Phenomena 23: 1883-1886.

4. Golberg D, Bando $Y$, Huang $Y$, Terao T, Mitome M, et al (2010) Boron Nitride Nanotubes and Nanosheets. AZO Nano 4: 2979-2993.

5. Chen Y, FitzGerald J, Williams JS, Bulcock S (1999) Synthesis of boron nitride nanotubes at low temperatures using reactive ball milling. Chemical Physics Letters 299: 260264.

6. Yu J, Chen Y, Wuhrer R, Liu Z, Ringer SP (2005) In Situ Formation of $\mathrm{BN}$ Nanotubes during Nitriding Reactions. Chem Mater 17: 5172-5176.

7. Loiseau A, Willaime F, Demoncy N, Hug G, Pascard H (1996) Boron nitride nanotubes with reduced numbers of layers synthesized by arc discharge. Phys Rev Lett 76: 4737-4740.
8. Terrones M, Hsu WK, Terrones H, Zhang JP, Ramos S, et al. (1996) Metal particle catalysed production of nanoscale BN structures. Chemical Physics Letters 259: 568-573.

9. Lourie OR, Jones CR, Bartlett BM, Gibbons PC, Ruoff RS, et al. (2000) CVD Growth of Boron Nitride Nanotubes. Chem Mater 12: 1808-1810.

10. RZ Ma, Y Bando, T Sato, K Kurashima (2001) "Growth, Morphology, and Structure of Boron Nitride Nanotubes". Chem Mater 13: 2965-2971.

11. JS Wang, VK Kayastha, YK Yap, ZY Fan, JG Lu, et al. (2005) Low Temperature Growth of Boron Nitride Nanotubes on Substrates. Nano Lett 5: 2528-2532.

12. Golberg D, Bando Y, Eremets M, Takemura K, Kurashima K, et al. (1996) Nanotubes in boron nitride laser heated at high pressure". Appl Phys Lett 69: 2045-2047.

13. Chen Y, Conway M, Williams JS, Zou J (2002) Large-quantity production of high-yield boron nitride nanotubes. Journal of Materials Research 17: 1896-1899.

14. Golberg D, Bando Y, Han W, Kurashima K, Sato T (1999) Single-walled B-doped carbon, B/N-doped carbon and BN nanotubes synthesized from single-walled carbon nanotubes through a substitution reaction. Chemical Physics Letters 308: 337-342.

15. Terauchi M, Tanaka M, Suzuki K, Ogino A, Kimura K (2000) Production of zigzag-type $\mathrm{BN}$ nanotubes and $\mathrm{BN}$ cones by thermal annealing. Chemical Physics Letters 324: 359-364.

16. Edwards RS, Coleman KS (2013) Graphene synthesis: relationship to applications. Nanoscale 5: 38-51.

17. http://proedgewire.com/graphite-graphene-intel/china-setsaccess-standards-for-its-graphite-industry/

18. Chakrabarti A, Lu J, Skrabutenas JC, Xu T, Xiao Z, et al. (2011) Conversion of CarbonDioxide to Few-layer Graphene. J Mater Chem 21: 9491-9493.

19. Park KT, Nersisyan HH, Chun BS, Lee JH (2011) Preparation of porous zirconium microspheres by magnesio thermic reduction and their microstructural characteristics. Journal of Materials Research 26: 2117-2122.

20. (2015) Technical report submitted to NASA for contract NNX12CA43C.

21. Khanam PN, Ponnamma D, AL-Madeed MA (2015) Electrical Properties of Graphene Polymer Nanocomposites, Graphene-Based Polymer Nanocomposites in Electronics, Springer Series on Polymer and Composite Materials. In: KK Sadasivuni, Springer International Publishing, Switzerland, 25-47.

22. (2015) Final Phase I Report for contract NNX15CG25P and its supplement. 